\title{
Repetition and trace interaction: Superadditivity
}

\author{
LAN BEGG and CRAIG GREEN \\ McMaster University, Hamilton, Ontario, Canada
}

\begin{abstract}
This paper reports five experiments that examined memory for repeated and unrepeated pairs of words. In over 40 experimental comparisons, cued recall of the repeated pairs was better than it would have been if the words had been repeated as independent cognitive events. Therefore, memory traces do interact with other traces of the same nominal items. Our account of superadditive recall is that some encodings fail on the final test because they lack a needed piece of information. Specifically, some need additional item-specific information to enable access by the cue, and some need relational information for recall of the target. The second trial is an implicit test of memory, whose results give the system a heuristic basis for standing pat or doing more encoding. If a retrieved encoding needs less than a new one to become a success, it has a good chance of becoming a success, and recall is superadditive. However, if the ease of retrieving the encoding is for the wrong reasons, such as massed repetition, the item remains a failure, and recall is subadditive.
\end{abstract}

Why does repetition help memory? Our answer is that the second occurrence of an item is an implicit memory test whose outcome is a heuristic comment about whether the item is adequately encoded. If the first encoding is retrieved easily (Tversky \& Kahneman, 1973) or fluently (Jacoby \& Dallas, 1981), the implication is that the item is adequately encoded already. If no encoding is retrieved, the item, like a new one, needs to be encoded to become adequate. The interesting case occurs if items retrieve the first encoding, but retrieval is sufficiently nonfluent or difficult that the item receives additional encoding. Because the additional encoding is influenced by the retrieved encoding, a dependency is established between the temporally distinct occasions.

Memory for repeated items has three sources. First, some items are encoded adequately after only one study. Second, some items that fail completely the first time become adequately encoded on the second trial. Third, some items are inadequately encoded for the test but are retrievable on the second trial. These items are primed, in the sense that the second encoding need not start from scratch. The extent to which repeated items exceed unrepeated items depends on how often each of these events occurs.

How much improvement should occur with repetition? A useful benchmark is the level of improvement that would occur if each trial adds an independent contribution to memorability (see Atkinson, Bower, \& Crothers, 1965 ). If the first trial adequately encodes some proportion, $x$, of the items and the second trial adds $x$ of the rest, then $x+x(1-x)$ repeated items will be remembered. If

\footnotetext{
The research was funded by Operating Grant A8122 from the Natural Sciences and Engineering Research Council of Canada to Ian Begg. Experiment 1 was part of an honors thesis by Craig Green. Many people offered comments and advice at various stages of the research, including Ann Anas, Arthur Glenberg, Reed Hunt, Andrea Snider, Endel Tulving, and Bruce Weaver. Reprints may be obtained from Ian Begg, Department of Psychology, McMaster University, Hamilton, ON L8S 4K1, Canada.
}

memory for repeated items is worse than expected, repetition is subadditive. If memory for repeated items is better than expected, repetition is superadditive. The question is whether the second trial adds less than $x, x$, or more than $x$ of the items that were missed the first time.

The fluency of retrieving the first encoding is a heuristic basis for assessing memorability. However, the less the second trial is like the test, the less faith can be placed in fluency as a predictor of success. If, for example, the second trial follows so soon after the first that all first encodings are fluently retrieved, poorly encoded items are unlikely to become adequate. There is much evidence that massed repetition has subadditive effects (Gartman \& Johnson, 1972; Glenberg \& Lehmann, 1980; Glenberg \& Smith, 1981; Jacoby, 1978; Paivio, 1974, 1975; Ross \& Landauer, 1978). If the trials are spaced, however, repeated items show a greater benefit. Many experiments have found that free recall of repeated items is additive (Glenberg \& Lehmann, 1980; Glenberg \& Smith, 1981; Paivio, 1974, 1975; Ross \& Landauer, 1978). Recognition, in contrast, is usually subadditive (Goldman \& Pellegrino, 1977; Ross \& Landauer, 1978) even with spaced repetition.

Thus repetition has additive effects on recall unless the ease of retrieving the first encodings lowers the odds that the items will benefit from a second chance. If the subjects encode items differently on the second occurrence, however, the ease of retrieving the first encoding no longer affects the odds. For example, Paivio $(1974,1975)$ found subadditive recall after massed repetition if the items were processed the same way each time. In contrast, changing the process, as in imaging a word and then pronouncing it, produced additive effects of massed repetition. Similarly, Gartman and Johnson (1972) found that changing the context for repeated items removed the effect of spacing and doubled recall.

In some conditions of the experiments we have reviewed, recall was superadditive. There are many other 
experiments in which recall of repeated items was too good to be additive (e.g., Craik \& Tulving, 1975; Gartman \& Johnson, 1972; Jacoby, Bartz, \& Evans, 1978; Mathews \& Tulving, 1973). However, as Watkins and Kerkar (1985) pointed out, there is no obvious difference between the cases in which recall is superadditive and the cases in which it is not.

In summary, free recall usually shows additive effects of repetition unless repetitions are massed and redundantly processed. Recognition is often subadditive even if repetitions are spaced. Although recall is usually additive, there are some reports of superadditive recall.

Why is there so little evidence for superadditive recall? One reason is that some artifacts inflate the predicted values. The expectation that the same proportion of items will be added on each trial overlooks the possibility that the items missed on the first trial are inherently difficult ones. If they are, then a low proportion of recall may be a consequence of the quality of the items rather than a consequence of repetition.

A second artifact is more subtle. Suppose one subject recalis .10 of unrepeated items and .19 of repeated ones, and another recalls .70 of unrepeated items and .91 of repeated ones. Each subject recalls the predicted proportion of repeated items. However, the mean recall of unrepeated items, .40 , predicts that the mean recall of repeated items should be .64 , which exceeds the actual mean of .55. Although each subject performs at the predicted level, the performance of the subjects as a group shows subadditive effects of repetition. Even calculating the predicted value for every subject and then averaging does not remove the problem, because the same overprediction comes about when we add over items within subjects.

Because of these artifacts, repetition is more effective than it appears to be from the published results. However, we need to consider an artifact that has the opposite effect: Recall of unrepeated items may suffer if repeated items are in the same list. Tulving and Hastie (1972) varied how many different words appeared in lists. At one extreme, all 15 words were different. At the other extreme, 7 words appeared twice and only 1 appeared once. Recall of unrepeated words was .43 , declining to .40 if there were 1 or 2 repeated words in the list, and to .37 if there were 6 or 7 . The decline is small (from .43 to .37 ), and so is the decline in predicted values (from .68 to .60 ). Note that recall of the repeated items also declined from .80 if there were 1 or 2 repeated words to .72 if there were 6 or 7 ; even the worst recall of repeated items exceeded the value predicted from the best recall of unrepeated items.

Waugh $(1962,1963)$ did not find inhibited recall of unrepeated items in lists that also contained repeated items. The discrepancy between results hinges on the procedures. Tulving and Hastie (1972) required their subjects to recall repeated words by writing them twice; Waugh did not. Hastie (1975) found inhibition of recall of unrepeated words only if subjects had to keep track of frequency.
However, even the best recall of unrepeated items underpredicted recall of repeated items $(.73<.77$, Experiment $1 ; .63<.72$, Experiment 2). Robbins, Bray, and Irvin (1974) examined recall of unrepeated pairs in lists containing repeated pairs, and also found inhibition only if subjects had to remember frequency. Again, recall of repeated pairs exceeded values predicted by the best recall of unrepeated pairs $(.64>.61$, Experiment 1; $.65>.55$, Experiment $2 ; .64>.53$, Experiment 3 ).

Thus recall of unrepeated items may suffer if repeated items are in the same list. If so, predicted values will be too low, increasing the odds of finding superadditive effects that are spurious.

Watkins and Kerkar (1985) used lists in which some items appeared once and others twice. In each of three experiments, they found superadditive recall of repeated items. In Experiment 2, for example, recall of repeated items was more than twice that of unrepeated items $(.44>$.17). However, recall of unrepeated items would need to improve from .17 to only .25 to predict recall of the repeated items. Free recall is a poor procedure when there are items of differential strength in the list. Inhibition of recall of weak items occurs if subjects recall the strong ones first (Roediger, 1974). Even modest inhibition could reduce recall from .25 to .17 .

Glenberg and Lehmann (1980) repeated items over lists. Immediately after the second list, subjects recalled .22 of the unrepeated items from the first list and .50 from the second. Recall of repeated items was .60 , which nearly equalled the predicted value, .61 . When the second test was delayed by a week, recall of repeated items was .26 , which exceeded the predicted value, .18. If the encodings were independent when they occurred, they would still be independent after a delay. Why, then, did recall become superadditive? After the delay, the repeated items were $43 \%$ of the immediate level, but the predicted value was only $30 \%$ of its former level. The items from the first list declined to $36 \%$ of their former level (.08) and those from the second list fell to $20 \%$ of their former level (.10). The predicted values are based on items that are poorly recalled. Even if true recall of the items was .10 from the first list and .18 from the second, the repeated items would be at the predicted value.

We do not know whether inhibition did occur in these experiments. However, even modest inhibition might have produced the results. In our own experiments, this was not a possibility.

By our account, the second trial is an implicit memory test whose outcome informs the system how much additional encoding is needed. With spaced repetition, the outcome of the test is correlated with success. Items whose first encodings are not retrieved will likely fail the final test. All other things equal, these items have the same chance of becoming adequate on the second trial as do new items under the same experimental conditions. Items whose encodings are adequate after one trial will likely be retrieved on the second trial; their fluent retrieval correctly implies that they need no additional encoding. All 
other things equal, the proportion of repeated items that are adequate after one trial will equal the proportion of unrepeated items from the first trial that ultimately succeed. If these two events are the only ones, memory for repeated items will be additive.

Memory can be superadditive only if three constraints are jointly met. First, some items must be inadequately encoded after one trial. Second, some of these inadequate encodings must be retrieved when the items are repeated; this constraint is likely to be met because the second trial is sooner than the test and it is more similar to the first trial than the test will be. Third, these encodings must be more likely than new items to become adequate. To be concrete, suppose that .40 of items are adequate after one trial and that half of the other .60 are retrieved on the second trial, at which time .70 become adequate. Recall is then .73 , which is only .09 better than the expected value, .64.

In summary, superadditive recall is rarely seen because there are artifacts working against it and because the difference between the observed and predicted values is a product of three probabilities, one of which is a difference. We will remove the artifacts in our experiments, and move from free recall to cued recall.

Why cued recall? We have not yet defined "adequacy of encoding." The organization-redintegration hypothesis (Begg, 1972, 1973, 1978a, 1982; Begg \& Sikich, 1984; Begg \& White, 1985) proposes that cued recall needs relational information and item-specific information. By that account, encoding is the process of interpreting items and organizing them into memory units. Items share a unit only if they are processed in relation to each other. Items are recognized only if they are distinctive within their units (Begg, 1978b; Jacoby, Craik, $\&$ Begg, 1979). Although the adequacy of the information that relates items is independent of the adequacy of the information that identifies each item, recall requires that both kinds of information be adequate. If a pair is encoded as two unrelated units, retrieval of one does not enable recall of the partner, and even a strong relation cannot mediate recall unless it is accessible by the cue.

Cued recall should show superadditive effects of repetition because of primed encodings for pairs that fail because only one of the two requisites is met. If pairs are retrieved on the second trial, they have a better chance than new pairs of becoming adequate. If the items are adequately related, but the future cue is inadequately encoded, the pair needs only additional encoding of that cue to be a success. Reciprocally, if the cue is recognizable, but unrelated to the target, the pair needs only the addition of relational information to be a success. New pairs need both requisites, and the odds of getting both are lower than the odds of getting one, because they are independent.

In summary, we have proposed that when a pair is studied, there is a chance that each item will acquire enough item-specific information for it to be recognized on the final test. Independent of this, there is a chance that the items will be related well enough that retrieval of one will allow recall of the other. After one trial, some pairs have both relational and item-specific information. These pairs will succeed regardless of what happens on a second trial. Some pairs have neither kind of information. These pairs will fail unless both kinds of information are encoded on the second trial. Other pairs have one kind of information but not the other. When repeated, these pairs retrieve the first encoding, but retrieval is less fluent than for pairs that retrieve fully adequate encodings. Nonfluent retrieval is a signal that more encoding is needed, and the retrieved encoding directs the course of the additional encoding. This cognitive crosstalk makes the second trial more effective than it would be if the two events were independent.

Although the theory is simple, it makes testable predictions. Cued recall will improve superadditively with repetition; however, recognition of single words will not, because it is more exclusively reliant on one factor, itemspecific information, than cued recall is. Furthermore, superadditive cued recall will occur whether the pairs are processed interactively or separately, because pairs lacking either kind of information are candidates for priming. Finally, superadditive cued recall will occur whether the pairs are processed in the same way or in a different way over trials; changing the process reduces the chance that fluent retrieval will interfere with the second encoding, but it also increases the chance that the first encoding will fail to be retrieved.

\section{OVERVIEW}

The rest of the article describes five experiments designed to determine whether memory for pairs increases superadditively with repetition. Because of the rarity of the phenomenon, we included many controls and conditions. In retrospect, most of these complications were unnecessary.

Our task can be symbolized as AB-BC. Some pairs (A) were in the first of two lists, some (C) were in the second, and others (B) were in both. Recall of the B pairs, $b$, was compared to the value predicted by $a+c(1-a)$. Memory was tested by cued recall in each experiment, and the pairs were studied by interactive or separate processes.

Experiment 1 used the $\mathrm{AB}-\mathrm{BC}$ sequence and two other ones, $A B-D C$ and $A D-B C$, in which another set of pairs, $D$, replaced one of the occurrences of the $B$ pairs. Each case gives a predicted value, $a+c(1-a)$, to compare with the repeated $B$ items. There is also a predicted value from the unrepeated B pairs: $b+b(1-b)$. The experiment allows very stringent comparisons between repeated and unrepeated items, the results of which indicate whether the unrepeated items are inhibited by the repeated ones.

The remaining experiments (2-5) used the $A B-B C$ paradigm. Experiments 2, 3, and 4 each compared four interactive conditions with four separate conditions. Within each, either both lists were studied the same way, 
imaginally or verbally, or one was imaginal and the other was verbal. The three experiments allow 24 comparisons between predicted and observed values for repeated items. Experiments 2, 3, and 4 included other comparisons as well. Experiment 2 included groups who studied single words rather than pairs, followed by free recall. Experiment 3 used longer lists and tested recognition of single words from the pairs. Experiment 5 studied within-list repetition. Interactive imagery again was contrasted with separate imagery, and the effects of repetition again were assessed for cued recall and for recognition.

Over the five experiments, there were more than 40 comparisons between cued recall of repeated items and its predicted value. In every comparison, the observed values exceeded the predicted ones. There were $16 \mathrm{com}-$ parisons for recognition: between-list repetition had additive effects, but within-list repetition was subadditive. There were also four comparisons for free recall: recall was superadditive, but barely.

\section{EXPERIMENT 1}

The purpose of Experiment 1 was to find out whether cued recall of repeated pairs exceeds the level expected if each presentation is an independent cognitive event. No previous research has asked this question of cued recall.

On the basis of previous research, we developed a paradigm in which the unrepeated items should not be inhibited by the repeated ones, but which allowed us to find out whether there is any inhibition, and to determine whether the recall of repeated items exceeds the level that would be predicted by unrepeated items without repeated items in the same list. The procedure was simple. Each subject studied two lists, each containing two sets of pairs. In the $\mathrm{AB}-\mathrm{BC}$ procedure, one set of pairs $(\mathrm{A})$ was in the first list, one set (C) was in the second, and one set (B) was in both. The two control procedures were $A B-D C$ and $\mathrm{AD}-\mathrm{BC}$, in which one of the occurrences of the $\mathrm{B}$ pairs was replaced by another set of pairs (D). If inhibition occurs, the $A$ and $C$ pairs should be recalled most poorly in the $A B-B C$ condition.

Superadditivity occurs if recall of repeated items exceeds the value predicted from recall of unrepeated pairs. Repeated items can be compared to $a+c(1-a)$, calculated for each of the three procedures. They can also be compared to $b+b(1-b)$, calculated from each of the control procedures in which the B items appeared only once. These contrasts are sufficiently stringent tests of superadditive recall because they rule out item selection and inhibition of unrepeated items.

\section{Method}

Subjects. One hundred eight students from McMaster University, in six groups of 18 , served to fulfill a requirement of the introductory psychology course.

Materials. Two hundred eight nouns with imagery values (Is) greater than 6 and familiarity values (Fs) greater than 10 were selected from Paivio, Yuille, and Madigan's (1968) word list. The nouns were sorted into eight sets of 26 , matched for mean imagery and familiarity values. The sets were assigned at random to be leftor right-hand members of four sets of pairs (A, B, C, D), and were paired at random. In the $\mathrm{AB}-\mathrm{BC}$ condition, List 1 had the $\mathrm{A}$ pairs and $B$ pairs randomly interspersed, and List 2 had the $B$ and $C$ pairs randomly interspersed; mean spacing for the two occurrences of the $B$ pairs was 52 (range $=35-70$ ) intervening pairs. In the AB-DC condition, the same List 1 was used, but the $D$ pairs replaced the $B$ pairs in List 2 . In the $A D-B C$ condition, the $D$ pairs replaced the $B$ pairs in List 1 .

Each List 1-List 2 sequence was recorded on videotape from a computer-generated display. The pair members appeared side by side, with $5 \mathrm{sec}$ from onset to onset. The test comprised the lefthand members from the $26 \mathrm{~A}$ pairs, the $26 \mathrm{~B}$ pairs, and the 26 $C$ pairs, in random order.

Procedure. All subjects were encouraged to follow instructions. The list presentation was described, and the subjects were told there might be repetitions. Half the subjects were given interactive imagery instructions, and half were given separate imagery instructions (Begg, 1978a); then the 104 pairs were presented. Following study, which required $8 \mathrm{~min}$, the subjects were tested by cued recall. The test was self-paced and took less than $10 \mathrm{~min}$.

\section{Results and Discussion}

Throughout this paper, all means are proportions and mean squared errors are squared proportions. The alpha level is .05 for inferences, including post hoc $t$ tests used to calculate critical differences for evaluating simple effects.

Proportionate recall of the 26 pairs of each set appears in Table 1. It is obvious that repetition did not inhibit recall of unrepeated pairs; mean recall of the $A$ and $C$ pairs in the $\mathrm{AB}-\mathrm{BC}, \mathrm{AB}-\mathrm{DC}$, and $\mathrm{AD}-\mathrm{BC}$ conditions was $.21, .20$, and .21 , respectively $(F<1)$. Recall of pairs having interactive imagery exceeded recall of those having separate imagery $[F(1,102)=51.8, M S e=0.0074]$, and recall of $\mathrm{C}$ pairs exceeded that of A pairs [.23 vs. $.19 ; F(1,102)=14.4, M S e=0.0069]$. For the unrepeated $B$ pairs, recall of pairs having interactive imagery exceeded recall of those having separate imagery $[F(1,68)$ $=35.8, M S e=0.016]$, and recall was unreliably better for pairs in List 2 than for those in List 1 (.21 vs. .18; $F=1.43$ ).

Table 1

Proportionate Cued Recall of Repeated and Unrepeated Pairs in Experiment 1

\begin{tabular}{|c|c|c|c|c|c|c|}
\hline \multirow[b]{2}{*}{ List Condition } & \multicolumn{3}{|c|}{ Unrepeated } & \multirow{2}{*}{$\frac{\text { Repeated }}{\text { B2 }}$} & \multicolumn{2}{|c|}{ Predicted By } \\
\hline & $\mathbf{A}$ & $\mathrm{C}$ & $\mathbf{B} 1$ & & $\mathrm{~A}, \mathrm{C}$ & B1 \\
\hline \multicolumn{7}{|c|}{ Separate Imagery } \\
\hline $\mathbf{A B}-\mathbf{B C}$ & .09 & .15 & - & .34 & .23 & - \\
\hline$A B-D C$ & .09 & .11 & .08 & - & .19 & .15 \\
\hline $\mathrm{AD}-\mathrm{BC}$ & .11 & .18 & .14 & - & .26 & .24 \\
\hline \multicolumn{7}{|c|}{ Interactive Imagery } \\
\hline$A B-B C$ & .26 & .34 & - & .62 & .49 & - \\
\hline AB-DC & .29 & .29 & .28 & - & .48 & .46 \\
\hline AD-BC & .27 & .29 & .29 & - & .47 & .48 \\
\hline
\end{tabular}

Note $-\mathrm{A}=$ pairs that were only in List $1, \mathrm{C}=$ pairs that were only in List $2, B 1=$ the B pairs that were in only one list, and B2 = the same pairs when they were in both lists. The prediction from $A, C=$ $a+c(1-a)$, and the prediction from $\mathrm{B} 1=b 1+b 1(1-b 1)$. 
It is also clear from Table 1 that recall of repeated B pairs exceeded all the predicted values. The recall of repeated pairs was first compared to the predicted values from the $\mathrm{A}$ and $\mathrm{C}$ pairs within subjects. Observed recall (.48) exceeded predicted recall $(.36)[F(1,34)=20.1$, $M S e=0.0092]$. Second, recall of repeated pairs was compared to values predicted from the $\mathrm{A}$ and $\mathrm{C}$ pairs in the two control lists. Again, recall exceeded the predicted values $(.33, .36)[F(2,102)=6.09, M S e=0.033$; a difference of .09 is reliable]. Finally, recall of repeated B pairs was compared to recall predicted by the unrepeated B pairs. Once again, recall exceeded the predicted values $(.30, .36)[F(2,102)=7.91, M S e=$ 0.036; critical difference $=.09$ ].

\section{Discussion}

As in previous research, there is no inhibition by repeated items on recall of unrepeated items if the subjects are not required to keep track of frequency. The demonstration of superadditive recall of repeated items against unrepeated items from lists containing no repeated items is a more stringent demonstration than the withinsubjects demonstrations. However, the point is the same: Recall of repeated items is better than expected if each repetition contributes independently to memorability.

\section{EXPERIMENTS 2, 3, AND 4}

The next three experiments used the $\mathrm{AB}-\mathrm{BC}$ procedure. Each subject studied two lists of word pairs; half the pairs in List 2 were new, and half were repetitions of pairs from List 1 . After both lists were presented, the left-hand members of all pairs were given as cues for recall of the righthand members. The contrast of interest is that between recall of repeated B pairs, $b$, and recall of unrepeated pairs, $a$ and $c$. Recall is superadditive if $b>a+c(1-a)$.

As in Experiment 1, subjects processed the two members of each pair separately or interactively. In addition, the encoding modality was varied; each list was encoded verbally or imaginally.

Encoding modality was manipulated because previous research has indicated that it matters whether items are encoded in the same way each time. Goldman and Pellegrino (1977) found additive recall for items processed a different way each time but superadditive recall for items processed the same way each time. Similarly, Thios (1972) repeated words in either the same or a different sentence context. Recall was subadditive $(.25<.34)$ if the repetitions were massed and in different contexts; spacing increased recall to .40 . However, recall of words repeated in the same context and massed was .40 ; spacing increased recall to .48 . Other research has shown better recall if items are repeatedly processed in the same context rather than in different contexts (Postman \& Knecht, 1983), in different orders (Polzella \& Chocinsky, 1974), or under different strategies (Young \& Bellezza, 1982).

Results of other research have differed, however. Superadditive recall was found in Paivio's $(1974,1975)$ studies only when repetitions were spaced and processed differently. Similarly, Gartman and Johnson (1972) found superadditive recall only if the contexts for the items were changed between repetitions. The strongest evidence for superadditive recall was reported by Watkins and Kerkar (1985), whose procedure included presenting the item in a different context on each occurrence. Thus varying or maintaining mode of processing over repetitions matters, but not in a simple way.

Experiments 2, 3, and 4 of this study differed from each other in some ways. In Experiment 2, free recall was tested after the subjects studied words rather than pairs. For stimuli that are single words, interitem relational processing should occur incidentally, if at all. We expected that free recall would be closer to being additive than would cued recall, because cued recall cannot succeed without both item-specific and relational information. In Experiment 3, longer lists were used and recognition was tested. We expected recognition to show additive effects of repetition. Goldman and Pellegrino (1977) found no cases in which recognition was superadditive, but they did find some cases in which recognition of repeated items was subadditive. No experiments to date have examined recognition of individual pair members in this context. In Experiment 4, shorter lists were used to improve performance with separately processed items. As we shall see, however, the procedural differences across experiments proved to be minor.

\section{Method}

Subjects. The subjects were 384 students of introductory psychology classes at McMaster University who served to fulfill a course requirement. In Experiment 2, eight groups of 20 studied pairs and four groups of 16 studied single words; in Experiment 3 , eight groups of 10 studied pairs; in Experiment 4, eight groups of 10 studied pairs.

Materials. The materials were nouns from Paivio et al.'s (1968) word list. In Experiment 2, 120 nouns (Is $\geq 6$ and $10<$ Fs $<99$ ) were used to make 60 pairs. In Experiment 3, 234 nouns (Is > 5 and $F s>26$ ) were used to make 78 pairs and 78 distractors for a recognition test. In Experiment 4, a random subset of 96 of the nouns from Experiment 3 was used to make 48 pairs. For each experiment the pairs were made the same way. For example, the 120 nouns in Experiment 2 were sorted into six sets of 20 that were nearly identical in mean imagery and frequency ratings. Random pairing between sets produced three sets of 20 pairs that were nearly identical in their normative characteristics. Similarly, for Experiment 3 there were three sets of 26 pairs, and for Experiment 4 there were three sets of 16 . Note that the items and their assignments to sets differed over the experiments.

In Experiment 2, each subject saw two lists of 40 pairs whose members were side by side on a television monitor. Pairs were presented at a rate of $10 \mathrm{sec}$ per pair. The monitor showed a videocassette recording of a computer-generated display. List 1 had the $20 \mathrm{~A}$ pairs and the $20 \mathrm{~B}$ pairs in random order. List 2 had the $20 \mathrm{~B}$ pairs and the $20 \mathrm{C}$ pairs in random order. Experiment 3 was the same except that there were 26 different pairs for A, B, and $C$, and Experiment 4 was also the same except that there were 16 different pairs. Thus, each experiment had an equal number of pairs that appeared only in List 1 (A pairs), only in List 2 (C pairs), or once in each list (B pairs).

Each experiment used a cued recall test on which the left-hand members of all the A, B, and $\mathrm{C}$ pairs were provided in random order, with a blank space next to each cue for recall of its partner. 
Experiment 2 also included lists of single words for the subject to study for $5 \mathrm{sec}$ each before the free recall test. The two lists of 40 words each comprised the right-hand members of the lists of pairs.

Experiment 3 included a recognition test consisting of the 78 distractors and 1 member of each of the 78 pairs; the tested item was equally often the left-hand and the right-hand member of a pair. The 156 words were in random order, with $\mathrm{Y}$ and $\mathrm{N}$ next to each one.

Procedure. The pairs in each experiment were studied under eight conditions resulting from the factorial combination of three variables. List 1 was studied verbally or imaginally, List 2 was studied verbally or imaginally, and both lists were studied interactively or separately. Instructions for imaginal interaction required that the subjects form a single image of the two members of each pair interacting in an imagined frame above the monitor. Instructions for imaginal separation required formation of a single image for each word in each pair, with each image projected to an imagined frame to the nearer side, left or right, of the monitor. Instructions for verbal interaction required the subjects to generate associates that were related to both words, and instructions for verbal separation required generation of associates for each word on its own. Associates were to be generated silently for as long as the pair was on the screen. Thus four groups studied the pairs interactively, and four studied each word separately. In each case, one group studied both lists verbally, one studied both lists imaginally, and two groups studied each list differently, one for each of the two orders. These eight conditions were used in each of the three experiments. The interval between lists was the $\mathbf{3 0} \mathrm{sec}$ needed for the instructions for List 2.

Immediately after study, the subjects in Experiments 2 and 4 were tested for cued recall, with 5 min allowed. The subjects in Experiment 3 were tested first for recognition $(7 \mathrm{~min})$ and then for cued recall (5 min).

The subjects in Experiment 2 who studied single words were in four groups. One group studied List 1 imaginally and List 2 verbally, a second group studied List 1 verbally and List 2 imaginally, a third group studied both lists imaginally, and the fourth group studied both verbally. After study, subjects had $5 \mathrm{~min}$ for free written recall.

\section{Results and Discussion}

Cued recall: Superadditive? The first question was whether the recall of repeated items was above the level predicted by assuming independence. For each subject, the value of $a+c(1-a)$ was calculated; the means shown in Table 2 are the averages of these values. Note that all $24 \mathrm{~B}$ values are larger than the predicted values. The difference was reliable in each experiment $[F(1,152)=$ $64.3, F(1,72)=136$, and $F(1,72)=57.1$, respectively] In Experiment 2, recall of the B items exceeded the predicted values less with interactive processing $(.68 \mathrm{vs}$. .61) than with separate processing (.43 vs. . 30$)[F(1,152)$ $=7.41 \mathrm{~J}$. In Experiment 3, the mean recall for the B items was .51 and the predicted value was .33, and there were no interactions. In Experiment 4, pairs studied twice by interactive imagery did not reliably exceed the predicted value, resulting in a four-way interaction $[F(1,72)=$ 4.52]; the interaction reflects a ceiling effect in this one comparison.

Cued recall of repeated pairs is well above the level predicted by assuming that each study occasion is an independent cognitive event. The only cases in which recall was not much higher than the predicted value were ones in which the unrepeated items were so well recalled that the predicted values were high. However, with a long list, in Experiment 3, all values exceeded the predicted values. In addition, superadditive recall occurred with interactive and separate processing, imaginal and verbal encoding, and repetition using the same or a different process.

Cued recall: Other results. The other results for cued recall will be presented briefly, because they are incidental to the present purposes. First, interactive processing led to better recall than did separate processing in all cases. The difference was reliable for the $A$ pairs, the $C$ pairs, and the $B$ pairs in each experiment. The smallest of the nine $F$ values was 36.3 .

Second, imaginal processing was generally better than verbal processing, but the difference was not as overwhelming as for interactive and separate processing. For the A pairs, there was a reliable main effect in each experiment; the smallest $F$ was 12.0. There were also some interactions. In Experiment 3, there was an interaction between interactive and separate processing and imagi-

Table 2

Mean Cued Recall for Unrepeated Items (A and C), Repeated Items (B), and the Values Predicted (Pr) for the Repeated Items

\begin{tabular}{|c|c|c|c|c|c|c|c|c|c|c|c|c|}
\hline \multirow[b]{2}{*}{ List 1-List 2} & \multicolumn{4}{|c|}{ Experiment 2} & \multicolumn{4}{|c|}{ Experiment 3} & \multicolumn{4}{|c|}{ Experiment 4} \\
\hline & $\bar{A}$ & $\mathrm{C}$ & B & $\operatorname{Pr}$ & $\mathrm{A}$ & $\mathrm{C}$ & B & $\overline{P r}$ & A & C & B & Pr \\
\hline \multicolumn{13}{|c|}{ Separate Processing } \\
\hline Verbal-Verbal & .11 & .20 & .40 & .27 & .07 & .09 & .38 & .15 & .08 & .26 & .42 & .31 \\
\hline Verbal-Imagery & .20 & .17 & .38 & 31 & .10 & .06 & .25 & .14 & .09 & .20 & .37 & .27 \\
\hline Imagery-Verbal & .23 & .11 & .42 & .31 & .11 & .00 & .21 & .11 & .12 & .16 & .36 & .26 \\
\hline Imagery-Imagery & .21 & .19 & .53 & .33 & .18 & .10 & .47 & .26 & .23 & .11 & .47 & .31 \\
\hline \multicolumn{13}{|c|}{ Interactive Processing } \\
\hline Verbal-Verbal & .24 & .30 & .51 & .44 & .29 & .26 & .65 & .43 & .40 & .56 & .87 & .72 \\
\hline Verbal-Imagery & .38 & .60 & .76 & .73 & .17 & .24 & .60 & .36 & .34 & .61 & .89 & .72 \\
\hline Imagery-Verbal & .48 & .32 & .70 & .59 & .44 & .20 & .71 & .54 & .41 & .43 & .84 & .63 \\
\hline Imagery-Imagery & .47 & .57 & .76 & .72 & .50 & .40 & .82 & .66 & .78 & .71 & .94 & .92 \\
\hline
\end{tabular}

Note-A = pairs that were only in List $1, \mathrm{C}=$ pairs that were only in List $2, \mathrm{~B}=$ pairs that were in both lists, and $\operatorname{Pr}=$ the predicted value for $\mathrm{B}$. $\mathrm{Pr}=a+c(1-a)$, calculated for each subject and averaged. MSe $<0.056$ for each column, and $M S e<0.013$ for contrasts between $B$ and Pr. 
nal and verbal processing $[F(1,72)=5.34]$ because the advantage for interactive over separate study was more pronounced with imaginal than with verbal processing. In Experiment 4, the advantage for the imaginally encoded pairs was especially great if the second list was also imaginally studied $[F(1,72)=11.1]$.

For $\mathrm{C}$ pairs in Experiment 2, imagery exceeded verbal processing $[F(1,152)=16.6]$, but more so in the interactive conditions than in the separate conditions $[F(1,152)=12.0]$. For $C$ pairs in Experiment 3 , there was only a slight advantage for imaginal over verbal processing $(.20$ vs. .14) $[F(1,72)=3.69, p=.06]$, but there was an interaction with how the first list had been processed $[F(1,72)=6.87]$; using the same procedure a second time was better than using a different one. For Experiment 4, imaginal study exceeded verbal study only in the interaction conditions $[F(1,72)=7.68]$.

Free recall. Mean free recall for the subjects in Experiment 2 who studied lists of single words is in the top section of Table 3 . For these words, the only reliable effect of processing condition was an interaction between imaginal and verbal processing and lists $[F(1,60)=4.01]$.

The repeated words were not recalled much better than was predicted. However, the small difference was reliable $[F(1,60)=7.63]$. Although there was no interaction over conditions, superadditivity occurred only in mixed conditions. In separate analyses of the four conditions, neither the verbal-verbal nor the imaginal-imaginal difference was reliable $[F(1,15)=1.27$, and $F(1,15)$ $=0.15$, respectively], but both the verbal-imaginal and the imaginal-verbal differences were $[F(1,15)=4.87$, and $F(1,15)=4.68$, respectively]. This weak finding favors theories that expect enhanced additivity with differential processing.

\section{Table 3}

Mean Free Recall of Single Words (Experiment 2) and Recognition of Single Members of Pairs (Experiment 3)

\begin{tabular}{llllll}
\hline & \multicolumn{2}{c}{ Unrepeated } & \multicolumn{2}{c}{ Repeated } \\
\cline { 2 - 6 } List 1-List 2 & $\mathrm{A}$ & $\mathrm{C}$ & $\mathrm{B}$ & $\mathrm{Pr}$ \\
\hline Experiment & $2:$ & Free & Recall of Single & Words \\
Verbal-Verbal & .27 & .27 & .49 & .47 \\
Verbal-Imagery & .30 & .26 & .52 & .47 \\
Imagery-Verbal & .28 & .27 & .54 & .47 \\
Imagery-Imagery & .32 & .36 & .58 & .56
\end{tabular}

Experiment 3: Recognition after Separate Study

$\begin{array}{lllll}\text { Verbal-Verbal } & .84 & .81 & .96 & .95 \\ \text { Verbal-Imagery } & .82 & .81 & .94 & .96 \\ \text { Imagery-Verbal } & .76 & .82 & .96 & .95 \\ \text { Imagery-Imagery } & .84 & .89 & .96 & .97\end{array}$

Experiment 3: Recognition after Interactive Study

\begin{tabular}{lllll} 
Verbal-Verbal & .75 & .74 & .92 & .93 \\
Verbal-Imagery & .69 & .72 & .90 & .91 \\
Imagery-Verbal & .78 & .69 & .92 & .91 \\
Imagery-Imagery & .81 & .79 & .95 & .95 \\
\hline
\end{tabular}

Note-A = pairs that were only in List $1, \mathbf{C}=$ pairs that were only in List $2, \mathrm{~B}=$ pairs that were in both lists, and $\mathrm{Pr}=$ the predicted value for $\mathrm{B} . \mathrm{Pr}=a+c(1-a)$, calculated for each subject and averaged. All MSes $<0.025$.
Recognition. Mean recognition in Experiment 3 is in the bottom two sections of Table 3 . Separate processing produced better recognition than did interactive processing, as it sometimes does (Begg, 1979, 1982, 1983; McGee, 1980). The advantage was marginal for A items (.81 and .76 for separate and interactive processing, respectively) $[F(1,72)=2.97, p=.09]$, but reliable for $C$ items $(.83>.73, F=12.1)$ and $B$ items $(.96>.92$, $F=5.61)$.

There was no evidence of superadditivity; the mean for repeated items (.939) was nearly identical to the predicted value (.941). The lack of difference between the observed and predicted values is not simply a ceiling effect, because the observed and predicted values track each other over the eight cells; indeed, $r=.87$, despite extreme attenuation of the range. Furthermore, the obtained values did have room to fall short of predicted values, but did not. Cautiously, we conclude that there is no evidence for trace interaction in recognition; privately, we think the results support the stronger conclusion of independence.

\section{Discussion}

Results of the three experiments all show that cued recall of repeated items is better than it would be if the two study occasions were independent cognitive events. Furthermore, repeated items exceed the predicted values for each study procedure, and if both trials use the same or a different procedure. With recognition and free recall, however, there is less evidence that would allow us to reject the assumption of independence.

In summary, pairs studied twice are particularly memorable. However, the value of repetition for memory differs across tests. Item-specific information, useful for recognition, may increase independently over study occasions. Cued recall also needs information relating the items. The full set of item-specific information and relational information increases more than expected on the second trial.

\section{EXPERIMENT 5}

The final experiment contrasted memory for repeated pairs with the level predicted by unrepeated pairs. A running list, in which some pairs occurred once and others twice, was used. Goldman and Pellegrino (1977) found that words repeated in a running list were recalled at or above the predicted level, but were recognized at or below the predicted level. Experiment 5 was designed to determine whether pairs would show the same pattern as words.

Two recognition tests were used. One was a yes-no test on which the subjects classified each item as new or old. The other was a frequency test on which the subjects estimated how often each item had occurred during study; recognition hits are estimates greater than zero. Proctor (1977) found better recognition inferred from frequency estimates than measured directly, but Harris, Begg, and 
Mitterer (1980) and Begg, Maxwell, Mitterer, and Harris (1986) did not.

The subjects studied the pairs using interactive or separate imagery. In addition, some subjects estimated frequency of occurrence of pairs at the time of study, to ensure attention to frequency. Other subjects judged whether each image was better or worse than the preceding one, using the same scale as those who estimated frequency.

\section{Method}

Subjects. Eighty introductory psychology students from McMaster University, with 10 in each of eight conditions, served to fulfill a course requirement.

Materials. One hundred ninety-two nouns (Is $>6.0$ and Fs $>$ 10) were selected from Paivio et al.'s (1968) word list. The nouns were sorted into six sets of 32 matched as closely as possible on imagery and frequency values. One set was selected at random for the 32 distractors on the test. Another set made 16 filler pairs. Two sets were left-hand items and two were right-hand items; random pairing gave two sets of 32 pairs, one to be unrepeated and one to be repeated.

The list comprised 120 pairs recorded on videotape from a computer-generated display in which pair members appeared side by side for $5 \mathrm{sec}$ each. There were five blocks of 24 pairs. Each block had the first occurrence of 16 pairs and the second occurrence of 8 . For the first block, all 8 second occurrences were fillers whose first occurrence was also in the block. Each succeeding block contained the second occurrence of 8 pairs whose first occurrence was in the preceding block, the first occurrence of 8 pairs whose second appearance was in the next block, and 8 pairs that were not repeated; the exception was the final block, in which 16 pairs had their only appearance. Thus, repeated items had a mean lag of 23 items intervening, with a range from 17 to 31 .

Two test booklets were constructed. Each contained the 32 lefthand members of unrepeated pairs, the 32 left-hand members of repeated pairs, and 32 new items. The 96 items were in four blocks of 24; each block had 8 new items, 8 unrepeated items ( 2 from each of the final four study blocks), and 8 repeated items ( 2 from each of the final four study blocks). To the right of each test item was a blank space for recall; to the left were $\mathrm{Y}$ and $\mathrm{N}$ for a recognition test, or 0,1 , and 2 for a frequency test.

Procedure. The list was shown to eight groups of subjects, al of whom had been asked to follow instructions and had been told of the nature of the list. Four groups had interactive imagery instructions, and four had separate imagery instructions. Each subject had a study sheet consisting of the numerals 1 to 120 , with a 0 and a 1 next to each. Two of each set of four groups were to circle 1 if they had imaged to the pair previously and 0 if they had not; these conditions are the on-line frequency conditions. The other subjects circled 1 if the present image was better than the immediately preceding one, and 0 if it was not. The task took about $10 \mathrm{~min}$.

Table 4

Mean Cued Recall on Recognition and Frequency Tests in Experiment 5

\begin{tabular}{lccc}
\hline & Unrepeated & Repeated & Predicted \\
\hline & Separate Imagery & \\
Recognition Test & .08 & .18 & .15 \\
Frequency Test & .06 & .17 & .12 \\
& Interactive Imagery & \\
Recognition Test & .19 & .42 & .32 \\
Frequency Test & .22 & .40 & .37 \\
\hline
\end{tabular}

Table 5

Mean Recognition Performance and Frequency Estimates in Experiment 5

\begin{tabular}{llll}
\hline \multicolumn{2}{c}{ Frequency of Occurrence } & $\begin{array}{c}\text { Recognition } \\
\text { (Predicted) }\end{array}$ \\
\hline 0 & 1 & 2 &
\end{tabular}

\begin{tabular}{lllll} 
On-line Goodness & .06 & .66 & .82 & .86 \\
On-line Frequency & .10 & .64 & .75 & .85 \\
& \multicolumn{5}{c}{ Frequency Test } \\
On-line Goodness & .15 & .71 & .80 & .90 \\
On-line Frequency & .32 & .71 & .84 & .89 \\
& \multicolumn{5}{c}{} \\
Frequency Estimates \\
On-line Goodness & 0.17 & 0.83 & 1.27 & \\
On-line Frequency & 0.36 & 0.87 & 1.30 & \\
\hline
\end{tabular}

Immediately after study, the test sheets were handed out, with the recognition test given to half of the subjects and the frequency test to half. The tests were self-paced and took less than $12 \mathrm{~min}$.

\section{Results and Discussion}

Cued recall. Mean recall values are in Table 4, averaged over the initial ratings of frequency or goodness, because that variable had no reliable effects. The first analysis contrasted recall of unrepeated pairs with recall of repeated pairs over the between-subjects conditions. Interactive study resulted in better recall than separate study $[F(1,72)=35.1, M S \mathrm{e}=0.0098]$, repeated pairs were recalled better than unrepeated pairs $[F(1,72)=151, M S e$ $=0.0064]$, and the two variables interacted $[F(1,72)=$ 19.2]; the effect of repetition was greater with interactive study $(.41$ vs. .20$)$ than with separate study $(.17$ vs. .07).

As in the other experiments, the comparison of interest is that between recall of repeated items and the level predicted by unrepeated items. Recall of repeated items exceeded the predicted values $[F(1,72)=19.0, M S e=$ 0.0061 , although the difference was less than in the discrete-list studies. The only interaction was the threeway interaction among imagery, test, and measure $[F(1,72)=5.16$, d. crit. $=.050]$; the eight means are in Table 4. Thus, as Goldman and Pellegrino (1977) found with single words, recall of repeated pairs is at or beyond the predicted level.

Recognition. Mean recognition scores are in the top two sections of Table 5; means are averaged over the two imagery instructions because they had no reliable effects. First, we considered the proportion of items called old over presentation frequencies of 0,1 , and 2 . The type of test $[F(1,72)=11.6, M S \mathrm{e}=0.0041]$ and frequency $[F(2,144)=828, M S e=0.011]$ had reliable main effects. Frequency interacted with set during study $[F(2,144)=8.88$, d. crit. $=.047]$; initial attention to frequency gave poorer recognition than did attention to image goodness, because of a higher false alarm rate in the new items $(.25>.09)$. Frequency also interacted with the type of test $[F(2,144)=6.99]$; more items were called old on the frequency test than on the recognition test for 
new items $(.24>.08)$ and unrepeated items $(.71>.65)$, but not repeated items (.82 vs. .78). Thus, old-new discrimination was better on the recognition test than on the frequency test.

Next, we compared recognition of repeated items to the level predicted by unrepeated items. As found by Goldman and Pellegrino (1977), recognition of repeated items was below the predicted level $[F(1,72)=44.5, M S \mathrm{e}=$ $0.0051]$. The only interaction was among measure, test, and set $[F(1,72)=7.91$, d. crit. $=.045]$; the eight means ar 2 in Table 5 . Recognition was reliably less than predicted except for the recognition test after goodness ratings, and even for that it was marginally less $(p=.06)$. Thus recognition shows subadditive effects of repetition.

Frequency estimates. Finally, we considered the frequency estimates, shown in the bottom panel of Table 5 , averaged over imagery. The only interaction was a marginal interaction between presentation frequency and online set $[F(2,72)=2.71, \mathrm{MSe}=0.032, p=.07$, d. crit. $=.11]$. Attention to frequency during study may have negatively affected accuracy.

\section{GENERAL DISCUSSION}

The results from the five experiments are easily summarized. Repetition has superadditive effects on cued recall but not on recognition of individual words. Superadditive recall is not an artifact of inhibition of unrepeated pairs by repeated pairs, nor do some otherwise potent variables affect whether recall of repeated pairs exceed the predicted values. Superadditive recall is a real phenomenon.

By our account, superadditivity occurs because traces interact within the cognitive system. After one trial, some pairs are already adequately encoded for a final test and some are not. The adequate ones do not need additional encoding. The ones that are not adequate, however, are not a unitary class. Some fail only because the cues are unrelated to the sought targets, some fail only because the cues cannot retrieve the unit that relates the cue and target, and others fail because there is no useful information in memory. Recall is additive if failures are a unitary class of poorly encoded items. It is the primed encodings that account for superadditivity. The second presentation of these pairs retrieves the earlier encodings, at which time only one of the two requisites for recall needs to be met to make these pairs recallable.

Our account places the cause of superadditive recall at the second encoding of repeated items. This account is formally indistinguishable from some other accounts. One could, for instance, propose that the dependency occurs at the time of the final test. A cue could retrieve a trace that is not itself adequate for memory, but that is adequate to enrich the cue, helping it to retrieve an adequate trace. The math is the same, and we have side-stepped this issue by the hedge "adequate for the final test." We can think of no way to distinguish these alternatives. Perhaps they are different in words only.
One could propose that dependency does not come about because the first encoding influences the second one, but rather because the second encoding causes retroactive recoding of the first one. Tulving (1983) described an experiment in which recall of BOY was contrasted under two conditions, namely BOY followed later in the list by GIRL, or BOY not followed by GIRL; the respective recall values were .60 and .34 . His explanation was that "at the time of the presentation of the word GIRL the subject 'thought back' to the earlier presentation of the word BOY, and that, as a result, the engram of BOY was recoded"' (p. 167). Tulving cited experiments by Loftus (e.g., Loftus \& Loftus, 1980) as additional support for the notion.

The difference between the conceptions is in the contents of the memory system. By our account, the system has (1) the trace of the first event, and (2) the trace of the second event as influenced by the retrieved trace of the first one. By the recoding account, the system has (1) the trace of the second event, and (2) the trace of the first event as recoded under the influence of the second one. For the BOY-GIRL example, Tulving has a BOY/GIRL trace and a GIRL trace, but we have a GIRL/ BOY trace and a BOY trace. Even if the two traces in our account are independent, recall of BOY would be $.34+.34(1-.34)=.56$, which is close to the .60 reported. It is not clear to us why the single BOY/GIRL trace in the recoding account is retrieved with a probability of .60 if a BOY trace had a probability of only .34 .

Recoding is a controversial possibility. McCloskey and Zaragoza (1985), for example, showed that the "recoding" in Loftus's work does not reflect loss of initial information, but rather reflects memory for new information added later. Similarly, Hasher, Attig, and Alba (1981) disconfirmed the notion that old information is "updated and erased" by new information. They showed that the initial information is recoverable if the subjects are later told to discount the information that "erased" the initial information. More generally, Martin (1971) considered the case in which people learn A-B pairs, then later learn A-C pairs. A-B recall declines as A-C improves, suggesting that the later occurrence of $C$ erased or knocked out $B$ from its association with $A$, or, in new words, the earlier A-B traces were recoded as A-C traces. Martin showed, however, that the ability of each $A$ to recall its $B$ partner was independent of its ability to recall $C$. The fact that recall increases or decreases over conditions does not logically imply that individual traces were recoded. Recoding is sufficient to produce differences between conditions, but it is not necessary; original traces could be intact but be harder to retrieve because later events add enough more "stuff" to the system to increase the amount of search needed, for example.

Our explanation is the neutral alternative; no theories deny that a current event can retrieve earlier ones, or that encoding is influenced by the current contents of the cognitive system. Recoding is a controversial alternative; it requires a demonstration that earlier traces are perma- 
nently altered. "To prove the recoding hypothesis wrong, therefore, all that is necessary is to show that all originally stored information is still functionally intact after recoding has taken place'" (Tulving, 1983, p. 168). We have no evidence in our experiments that original information is intact. However, each time the recoding account has appeared in a new disguise, some investigator has found that the old stuff is still there. We fear the same sequence will occur whenever the literature is ignored, because recoding is a cute possibility, even if it is wrong.

In summary, we propose that superadditive recall occurs because some encodings that are inadequate for recall are fertile ground in which to do additional encoding. Such encodings have enough item-specific information for recognition of the cue or enough relational information binding the cue and target, but not both. Item-specific information on its own, however, does not increase more from a second study occasion than expected on the basis of independence, as judged by recognition of repeated items. Thus relational information and item-specific information are influenced by factors that are independent of each other (cf. Humphreys, 1978; Hunt \& Einstein, 1981), even though the two kinds of information interact in the course of successful recall. Even if our explanation is wrong, however, superadditive recall is a real phenomenon, and it needs some explanation.

\section{REFERENCES}

Atkinson, R. C., Bower, G. H., \& Crothers, E. J. (1965). An introduction to mathematical learning theory. New York: Wiley.

BEGG, I. (1972). Recall of meaningful phrases. Journal of Verbal Learning \& Verbal Behavior, 11, 431-439.

BEGG, I. (1973). Imagery and integration in the recall of words. Canadian Journal of Psychology, 27, 159-167.

BEGG, I. (1978a). Imagery and organization in memory: Instructional effects. Memory \& Cognition, 6, 174-183.

BEGG, I. (1978b). Similarity and contrast in memory for relations. Memory \& Cognition, 6, 509-517.

BEGG, I. (1979). Trace loss and the recognition failure of unrecalled words. Memory \& Cognition, 7, 113-123.

BEGG, I. (1982). Imagery, organization and discriminative processes Canadian Journal of Psychology, 36, 273-290.

BEGG, I. (1983). Imagery instructions and the organization of memory. In J. C. Yuille (Ed.), Imagery, memory and cognition: Essays in honor of Allan Paivio (pp. 91-116). Hillsdale, NJ: Erlbaum.

BegG, I., Maxwell, D., Mitterer, J. O., Harris, G. (1986). Estimation of frequency: Attribute or attribution? Joumal of Experimental Psychology: Learning, Memory, \& Cognition, 12, 496-508.

BEGG, I., \& SIKICH, D. (1984). Imagery and contextual organization. Memory \& Cognition, 12, 52-59.

BEGG, I., \& WHITE, P. (1985). Encoding specificity in interpersonal communication. Canadian Journal of Psychology, 39, 70-87.

Craik, F. I. M., \& Tulving, E. (1975). Depth of processing and the retention of words in episodic memory. Journal of Experimental Psychology: General, 104, 268-294.

Gartman, L. M., \& Johnson, N. F. (1972). Massed versus distributed repetition of homographs: A test of the differential-encoding hypothesis. Journal of Verbal Learning \& Verbal Behavior, 11, 801-808.

GlenderG, A. M., \& LehmanN, T. S. (1980). Spacing repetitions over 1 week. Memory \& Cognition, 8, 528-538.

GlenberG, A. M., \& SMITH, S. M. (1981). Spacing repetitions and solving problems are not the same. Journal of Verbal Learning \& Verbal Behavior, 20, 110-119.

Goldman, S. R., \& Pellegrino, J. W. (1977). Processing domain, encoding elaboration and memory trace strength. Journal of Verbal Learning \& Verbal Behavior, 16, 29-44.

Harris, G., BeGG, I., \& MitTERER, J. O. (1980). On the relation between frequency estimates and recognition memory. Memory \& Cognition, 8, 99-104.

Hasher, L., Attig, M. S., \& Alba, J. W. (1981). I knew it all along: Or, did I? Journal of Verbal Learning \& Verbal Behavior, 20, 86-96. HASTIE, R. (1975). Intralist repetition in free recall: Effects of frequency attribute recall instructions. Journal of Experimental Psychology: Human Learning \& Memory, 104, 3-12.

HuMPHREYS, M. S. (1978). Item and relational information: A case for context independent retrieval. Journal of Verbal Learning \& Verbal Behavior, 17, 175-187.

Hunt, R. R., \& Einstein, G. O. (1981). Relational and item-specific information in memory. Joumal of Verbal Learning \& Verbal Behavior, 20, 497-514.

$\mathrm{J}_{\mathrm{ACOBY}}$, L. L. (1978). On interpreting the effects of repetition: Solving a problem versus remembering a solution. Joumal of Verbal Leaming \& Verbal Behavior, 17, 649-668.

JACOBY, L. L., BARTZ, W. H., \& Evans, J. D. (1978). A functional approach to levels of processing. Journal of Experimental Psychology: Human Learning \& Memory, 4, 331-346.

JACOBY, L. L., CRAIK, F. I. M., \& BEGG, I. (1979). Effects of decision difficulty on recognition and recall. Journal of Verbal Learning \& Verbal Behavior, 18, 585-600.

JACOBY, L. L., \& DALLAS, M. (1981). On the relationship between autobiographical memory and perceptual learning. Journal of Experimental Psychology: General, 110, 306-340.

LofTUS, E. F., \& LofTUS, G. R. (1980). On the permanence of stored information in the human brain. American Psychologist, 35, 409-420.

MARTIN, E. (1971). Verbal learning theory and independent retrieval phenomena. Psychological Review, 78, 314-332.

MATHEws, R. C., \& TUlVING, E. (1973). Effects of three types of repetition on cued and noncued recall of words. Journal of Verbal Learning \& Verbal Behavior, 12, 707-721.

McCloskey, M., \& Zaragoza, M. (1985). Misleading postevent information and memory for events: Arguments and evidence against memory impairment hypotheses. Journal of Experimental Psychology: General, 114, 3-18.

MCGEe, R. (1980). Imagery and recognition memory: The effects of relational organization. Memory \& Cognition, 8, 394-399.

PaIvio, A. (1974). Spacing of repetitions in the incidental and intentional free recall of pictures and words. Journal of Verbal Learning \& Verbal Behavior, 13, 497-511.

PaIvio, A. (1975). Coding distinctions and repetition effects in memory. In G. H. Bow'er (Ed.), The psychology of learning and motivation (Vol. 9). New York: Academic Press.

Paivio, A., Yullle, J. C., Madigan, S. A. (1968). Concreteness, imagery, and meaningfulness values for 925 nouns. Journal of Experimental Psychology Monograph Supplement, 76(1, Pt. 2).

Polzella, D. J., \& Chocinsky, R. C. (1974, May). Free recall: $A$ levels-of-processing interpretation. Paper presented at the meeting of the Midwestern Psychological Association, Chicago.

Postman, L., \& KNECHT, K. (1983). Encoding variability and retention. Journal of Verbal Learning \& Verbal Behavior, 22, 133-152.

Proctor, R. W. (1977). The relationship of frequency judgments to recognition. Journal of Experimental Psychology: Human Learning \& Memory, 3, 679-689.

RobBins, D., BRAY, J. F. , IRvin, J. R. (1974). Intralist contrast effects in cued recall. Joumal of Experimental Psychology, 103, 150-155.

RoEDIGER, H. L., III. (1974). Inhibiting effects of recall. Memory \& Cognition, 2, 261-269.

Ross, B. H., \& Landauer, T. K. (1978). Memory for at least one of two items: Test and failure of several theories of spacing effects. Journal of Verbal Learning \& Verbal Behavior, 17, 669-680. 
THIos, S. J. (1972). Memory for words in repeated sentences. Journal of Verbal Learning \& Verbal Behavior, 11, 789-793.

Tulving, E. (1983). Elements of episodic memory. New York: Oxford University Press.

TulviNG, E., HASTIE, R. (1972). Inhibition effects of intralist repetition in free recall. Joumal of Experimental Psychology, 92, 297-304.

TVersky, A., \& KaHneman, D. (1973). Availability: A heuristic for judging frequency and probability. Cognitive Psychology, 5, 207-232.

WATKINS, M. J., \& KeRKAR, S. P. (1985). Recall of a twice-presented item without recall of either presentation: Generic memory for events. Joumal of Memory \& Language, 24, 666-678.
WAUGH, N. C. (1962). The effect of intralist repetition on free recall. Journal of Verbal Learning \& Verbal Behavior, 1, 95-99.

WAUGH, N. C. (1963). Immediate memory as a function of repetition. Journal of Verbal Leaming \& Verbal Behavior, 2, 107-112.

YounG, D. R., \& BellezzA, F. S. (1982). Encoding variability, memory organization, and the repetition effect. Journal of Experimental Psychology: Learning, Memory \& Cognition, 8, 545-559.

(Manuscript received December 19, 1985; revision accepted for publication August 26, 1987.) 